\title{
Calcium phosphate neuron-like structures: a rare case or a common structure?
}

Cite this: J. Mater. Chem. B, 2014, 2 , 2020

\begin{abstract}
M. Espanol, ${ }^{a b c}$ Z. T. Zhao, ${ }^{\text {abc }}$ J. Almunia ${ }^{a}$ and M.-P. Ginebra*abc
Underneath the unique and beautiful structures of biominerals there is always the presence of organic molecules that tightly interact with the developing inorganic nuclei/crystal directing its growth and assembly towards the final structure. This close interdigitation between organic and inorganic matter renders biominerals not only unique in their appearance but also with exceptional properties. A notable case of such combination is observed when combining double hydrophilic block copolymers (DHBCs) with different ions. In the particular case of calcium phosphate systems, the incorporation of DHBCs was found to induce the formation of unique and delicate neuron-like structures. The present article highlights that such structures are more common than probably expected and they can be created using much simpler organic molecules of a wider nature such as non-ionic surfactants (Tween 80 or Span 20), anionic polymers (sodium polyacrylate) and cationic polymers (polydiallyldimethylammonium chloride). The reaction conditions are however crucial in the stabilization of the structures.
\end{abstract}

Received 29th October 2013

DOI: $10.1039 / c 3 t b 21524 \mathrm{e}$

www.rsc.org/MaterialsB the early stages of mineralization is a real challenge and though some studies point to the presence of this phase, ${ }^{6,7}$ until now there is no irrefutable proof of its presence. ${ }^{8,9}$ Dealing with amorphous phases is a complex task owing to the unstable nature and continuous transformation that such phases undergo; however, they offer the most remarkable morphologies found in nature. Amorphous phases, being isotropic, have no preferred form and can be easily molded/assembled into any shape with the help of organic structure directing molecules., ${ }^{2,10}$

Several kinds of organic additives ${ }^{11-16}$ have been used for the control of nucleation and crystal growth of inorganic materials but among them double hydrophilic block copolymers (DHBCs) have been found most effective in the crystallization of various materials. ${ }^{16-19}$ Particularly in the calcium phosphate system, Antonietti et al. reported back in 1998 the stabilization of delicate neuron-like calcium phosphate structures with the help of a DHBC consisting of a long poly(ethylene oxide) block and a short poly(methacrylic acid) block partially alkylated with dodecylamine (PEO-block-PMMA-C12). ${ }^{20}$ These structures were again replicated by Tjandra et al. in 2005 using a similar design of the DHBC (PEO-block-PMMA) but with a longer PMMA chain. ${ }^{21}$ The need for the complex DHBC seemed mandatory for the formation of such structures, and the mechanism of formation was believed to occur via formation of micelle aggregates of the copolymer. This conclusion was taken from the observation that the core of the neuron-like structures was of the same size as that of the primary polymer micelle aggregates. In the work of Antonietti, micelles could be readily created in the presence of the hydrophobic C12 chain, and in the work of Tjandra, micelle formation was believed to take place by neutralization of the carboxyl groups in the longer PMMA chain by calcium ions, rendering the PMMA-Ca chain 
hydrophobic. Surprisingly, in 2001 the work by Peytcheva et al. ${ }^{22}$ showed that polyaspartate was also capable of creating similar structures under specific synthesis conditions but no details were given with regard to the need for forming aggregates.

This article is a summary of recent investigations in our group where we have discovered that we can create identical neuron-like structures using very simple organic molecules of a wide nature thus bringing renewed interest in understanding the mechanism that governs their formation. Although we are yet far away from giving an exact explanation as to how they form, we will point to crucial aspects which are required to ensure their stabilization. We believe that the poor specificity existing with regard to the organic phase in these particular structures makes them particularly interesting in the biomineralisation field as all ingredients required for their formation are readily available in the body fluid. Moreover, the present results might shed new light on the creation of a new class of materials with additional functional properties.

\section{Experimental}

\subsection{Materials}

Unless otherwise stated, all materials were purchased from Sigma and used without further purification. Sodium polyacrylate with a molecular weight of $1200 \mathrm{~g} \mathrm{~mol}^{-1}$ and polydiallyldimethylammonium chloride of 100 000-200000 $\mathrm{g} \mathrm{mol}^{-1}$ were chosen for the present study. Calcium chloride was purchased from Panreac. For clarity purposes all organic molecules employed in the work are summarized in Table 1.

\subsection{Calcium phosphate precipitation in the presence of surfactants}

Stock solutions of $10 \mathrm{mM} \mathrm{CaCl}_{2}$ and $\mathrm{Na}_{2} \mathrm{HPO}_{4}(\mathrm{pH}$ 7.2-7.4) were used in all experiments. A typical reaction consisted of mixing equal volumes of both salts to make reactions with a calcium to phosphorous $(\mathrm{Ca} / \mathrm{P})$ molar ratio of 1 . This ratio was chosen to allow comparison of the present results with those available from the literature. ${ }^{11}$ The effect of TW was investigated by adding different amounts of surfactant into the calcium chloride solution (mixing $20 \mathrm{~min}$ ) prior to addition of the phosphate

Table 1 List of organic molecules along with their chemical formula

Tween 80 (TW) polysorbate 80

Span 20 (SP) sorbitan monolaurate

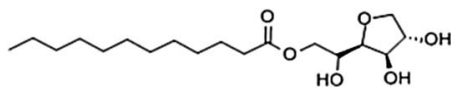

Sodium polyacrylate (PA)

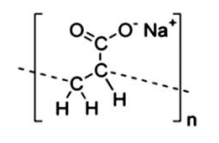

Polydiallyldimethylammonium chloride (PDMAC) salt into the mixture (phosphate solution was added at once). The concentrations of tween explored ranged from 0.01-3.3 wt/v $\%$ in the final mixture. Reactions were carried out in polypropylene tubes at a constant temperature of $25{ }^{\circ} \mathrm{C}$ and under magnetic stirring. The temperature was controlled by means of thermojacketed vessels coupled to a thermostatic bath (Huber CC2). Aside from exploring the effect of TW, the temperature of the reaction at 37 and $60{ }^{\circ} \mathrm{C}$ and also the addition of other types of surfactants such as Span 20 (SP) at the final concentration range of $0.01-0.3 \mathrm{wt} / \mathrm{v} \%$ were also investigated. Characterisation is comprised of $\mathrm{pH}$ and conductivity monitoring (Crison 5014 and MM41 respectively), transmission electron microscopic observation and EDX analyses (Jeol JEM 2100, Jeol JEM 1010 and Hitachi $800 \mathrm{MT}$ ) and field emission scanning electron microscopic observation (FIB/SEM Zeiss Neon 40). Samples for TEM examination were prepared by soaking a 300 mesh carboncoated copper grid in the solution of interest, blotted to remove the excess liquid and air dried. SEM examination was performed directly from the TEM grids without any kind of coating.

\subsection{Calcium phosphate precipitation in the presence of sodium polyacrylate}

Synthesis reactions incorporating sodium polyacrylate (PA) were carried out in a similar manner as those reported with the addition of surfactants. The reaction protocol consisted of mixing first the calcium chloride solution with PA (mixing 20 min) prior to addition of the phosphate solution. Various additional synthesis conditions were investigated: (1) two different calcium to phosphorous ratios were explored $\mathrm{Ca} / \mathrm{P}=1$ and $1.67,(2)$ for the $\mathrm{Ca} / \mathrm{P}=1.67$, different synthesis reactions were performed with increasing amounts of calcium concentrations with final molarities of $\sim 5,10$ and $50 \mathrm{mM}$, (3) two different concentrations of PA were explored to make $\mathrm{Ca} / \mathrm{PA}$ molar ratios of $\sim 20$ and 80 and (4) the effect of $\mathrm{pH}$ during the synthesis reaction was investigated by pre-adjusting the $\mathrm{pH}$ value of the calcium and phosphate salts as well as that of the PA solution to the pH of interest: $\mathrm{pH} \mathrm{3,} 7$ or 10. This was achieved using $\mathrm{NaOH}$ or $\mathrm{HCl}$. Stock solutions of $100 \mathrm{mM} \mathrm{CaCl}_{2}, 100$ $\mathrm{mM} / 60 \mathrm{mM} \mathrm{Na} \mathrm{HPO}_{4}$ and $1 \mathrm{wt} \%$ PA $(\sim 10 \mathrm{mM})$ were prepared and the required volumes were mixed to carry out all the above mentioned synthesis reactions. Characterisation of the reactions and products was done as specified in the preceding section. TEM grids were prepared from the supernatant within 2-5 $\mathrm{h}$ after reaction.

The interaction of PA with calcium ions was evaluated by titration: (1) a $10 \mathrm{mM}$ PA solution was added dropwise, at a rate of $0.5 \mathrm{ml} \mathrm{min}{ }^{-1}$ and under constant temperature $\left(25^{\circ} \mathrm{C}\right)$ and stirring, into $15 \mathrm{ml}$ of a $10 \mathrm{mM} \mathrm{CaCl}_{2}$ solution and (2) a $10 \mathrm{mM} \mathrm{CaCl}_{2}$ solution was added dropwise, at a rate of $0.5 \mathrm{ml} \mathrm{min}^{-1}$ and under constant temperature $\left(25^{\circ} \mathrm{C}\right)$ and stirring, into $15 \mathrm{ml}$ of a $2 \mathrm{mM}$ PA solution. Titrations were performed at $\mathrm{pH} \mathrm{3,7}$ and 10.

\subsection{Calcium phosphate precipitation in the presence of polydiallyldimethylammonium chloride}

Syntheses with polydiallyldimethylammonium chloride (PDMAC) were carried out by mixing the phosphate salt solution with the 
cationic polymer first, prior to addition of the calcium salt. For this purpose, equal volumes of $60 \mathrm{mM} \mathrm{Na}_{2} \mathrm{HPO}_{4}$ salt of $\mathrm{pH} 7.4$ and $100 \mathrm{mM} \mathrm{CaCl}_{2}$ solution were used $(\mathrm{Ca} / \mathrm{P}=1.67)$ and two different PDMAC concentrations, to make a final concentration of 0.1 and $0.001 \mathrm{wt} \%$, were investigated. Characterisation of the products was performed as reported previously. TEM grids were prepared directly from the suspension within $2-5 \mathrm{~h}$ after reaction.

\section{Results and discussion}

\subsection{The calcium phosphate precipitation system}

The first aspect that has to be considered when studying nucleation and mineralization events is the design of the system, i.e. the reaction conditions. Although the term biomineralisation already narrows down experiments to those taking place in aqueous environments under mild temperatures and $\mathrm{pH}$, the reactant concentration was carefully chosen in the present work so as to enable visualization of the amorphous to crystalline transition on an adequate time frame. Fig. 1 depicts the typical $\mathrm{pH}$ and conductivity profiles over time obtained when mixing equal volumes of a $10 \mathrm{mM}$ calcium salt solution with a $10 \mathrm{mM}$ phosphate salt solution previously adjusted to physiological pH. Time zero corresponds to the time point at which the phosphate salt solution was added into the calcium solution. Both, the $\mathrm{pH}$ and conductivity profiles are alike and, in
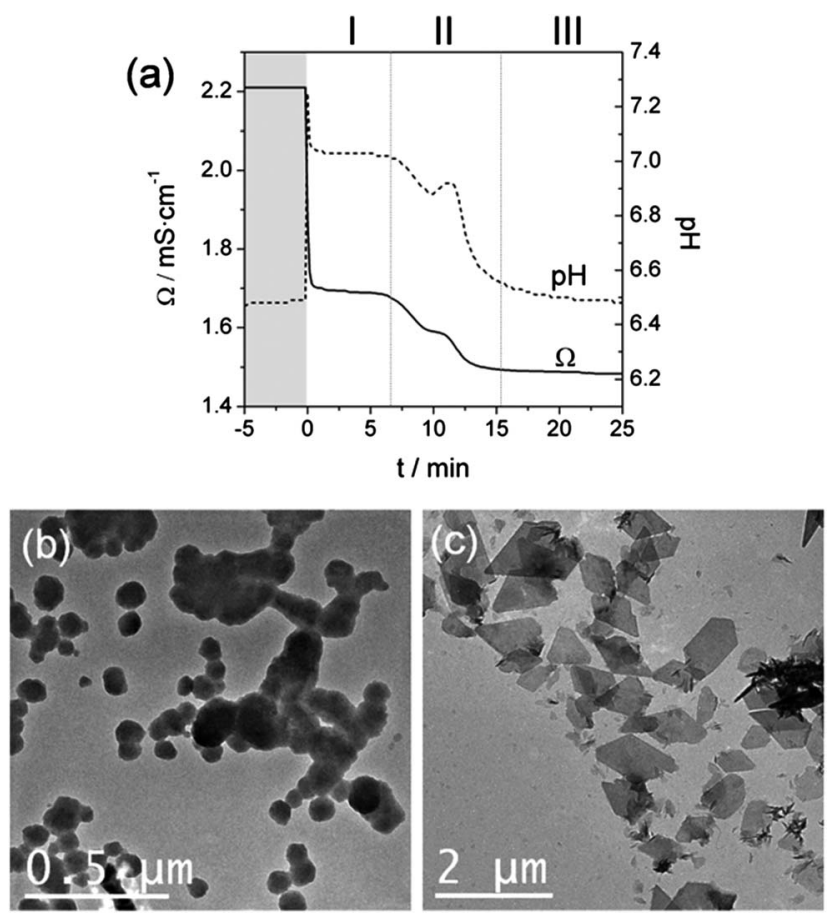

Fig. 1 (a) $\mathrm{pH}$ and conductivity profile of a typical synthesis reaction. Before time zero corresponds to the monitoring of a $15 \mathrm{ml}$ of $10 \mathrm{mM}$ calcium chloride solution. At time zero an equal volume of a $10 \mathrm{mM}$ phosphate solution ( $\mathrm{pH}$ 7.4) is added at once. (b) TEM image of the precipitates obtained within the amorphous regime in region I, i.e. 5 min after phosphate addition. (c) TEM image of the crystallized product from region III. agreement with other reported studies, they can be described by three distinct regions. ${ }^{11}$ The first region (I), immediately after mixing of both salts, consists of a plateau and corresponds to the region where amorphous calcium phosphate (ACP) exists, this is followed by a steep decrease in $\mathrm{pH} /$ conductivity (region II) which depicts the transformation of the amorphous to the crystalline phase. This transition involves the temporary stabilization of another amorphous phase as observed from the hump in the $\mathrm{pH}$ and conductivity profile. The third region (III) is a plateau and corresponds to the final reaction stage, the crystallized product. Such phase transition events are typical of kinetically driven crystallization processes and involve the continuous precipitation of phases of increasing stability. The macroscopic observation of the reaction starts with an almost transparent solution in region I that becomes turbid at region III corresponding to the bulk precipitation of calcium phosphate (CaP) crystals. Transmission electron microscopy (TEM) grids prepared from regions I and III confirmed the presence of globular ACP (these are structures which are easily damaged upon electron irradiation) and CaP crystals respectively (Fig. 1).

\subsection{Calcium phosphate precipitation in the presence of surfactants}

The addition of different amounts of a non-ionic surfactant Tween 80 (polysorbate 80, TW) into the calcium solution before mixing the phosphate salt revealed interesting results (Fig. 2). In terms of evaluation of the conductivity profiles, similar
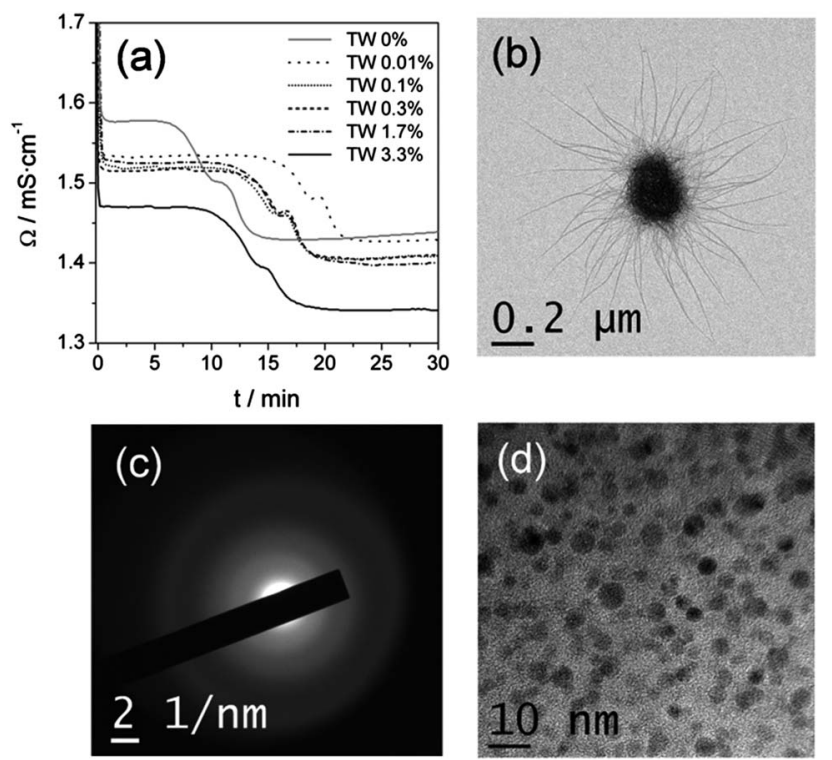

Fig. 2 (a) Effect of the addition of various contents of Tween 80 (TW) in the conductivity profile. TW was first allowed to mix with the calcium chloride solution before the phosphate solution was added at time zero. The concentrations of the salts were identical as in Fig. 1. (b) TEM image obtained for a TW content of $0.1 \mathrm{wt} \%$ after $5 \mathrm{~min}$ of addition of the phosphate salt. (c) Selected area electron diffraction (SAED) of the neuron-like structure revealing diffuse rings which accounts for the amorphous nature of these structures. (d) TEM image obtained for a TW content of $1.7 \mathrm{wt} \%$ after $5 \mathrm{~min}$ of addition of the phosphate salt. 
curves were obtained thus suggesting that the same events took place regardless of whether surfactant was added or not. A delay in the transition from ACP to the crystalline phase was the only distinct feature aside from the fact that this delay was more pronounced with a lower TW content. Such observations have also been reported using different molecules and the delay has been explained by the interaction of the organic molecules with the nascent nuclei. ${ }^{\mathbf{1 1}, 12}$ Although the impact of the addition of TW on the conductivity profile was very discreet, TEM investigation of the microstructures formed within the amorphous region was remarkable. For a TW content $\leq 0.1 \mathrm{wt} \%$, delicate calcium phosphate neuron-like structures with a $\mathrm{Ca} / \mathrm{P}$ ratio of $\sim 1$ as revealed by EDX analyses were formed along with the typical globular amorphous structures (Fig. 1). In addition to EDX, EELS analyses were also performed confirming the presence of $\mathrm{Ca}$ and $\mathrm{P}$ in them. What was most surprising was the fact that unlike for the globular precipitates, the neuron-like structures did not disappear upon amorphous to crystalline transformation and even after 5 months such structures persisted, keeping their amorphous nature. Increasing the amount of TW above $0.1 \mathrm{wt} \%$ caused the stabilization, within the amorphous region, of tiny rounded precipitates $(<5 \mathrm{~nm})$ which were presumably the initial amorphous $\mathrm{CaP}$ before they began aggregating to form larger globular particles. Thus, taking all these results altogether we can distinguish two different mechanisms of TW interactions: (a) for a low amount of TW the surfactant acts as a structure directing agent leading to formation of calcium phosphate neuron-like structures; but (b) an increase in the TW content quenches the surface of the initial amorphous nuclei delaying their subsequent growth/assembly.

Although the formation of these neuron-like structures bears some resemblance to the structures isolated during transformation of ACP to hydroxyapatite (HA) in purely inorganic systems, ${ }^{23}$ it is worth stressing that different mechanisms are likely to operate. In the crystallisation process of HA, ACP serves as a seed in the nucleation of the crystalline phase. Instead, if we combine our results with those of Antonietti et al. ${ }^{20}$ and Gower et al. ${ }^{10}$ we could think of the formation of the neuron-like structures as the transformation of an inorganic/organic hybrid core consisting of amorphous micellar precursors (with a look identical to that of "ACP", Fig. 1a). This would be followed by the secondary nucleation of branches due to the limited feeds of reactants. Although this mechanism needs further investigation, the sequence of events proposed have been isolated from a synthesis reaction at the early stages of precipitation (i.e. $5 \mathrm{~min}$ after mixing of all reactants) and the most representative images are compiled in Fig. 3. The amorphous nature of these structures was proved through SAED and HR-TEM analyses. HRTEM in particular showed that both the core and filaments of the structures were amorphous as no lattice fringes could be detected. It is important to mention that during HR-TEM, these particular structures (the well-formed ones, Fig. 3d) were not damaged which could otherwise cause misinterpretation of the results.

Interestingly, performing similar experiments at 37 and $60{ }^{\circ} \mathrm{C}$ led to considerable changes in the conductivity profiles but this did not prevent formation of the neuron-like structures
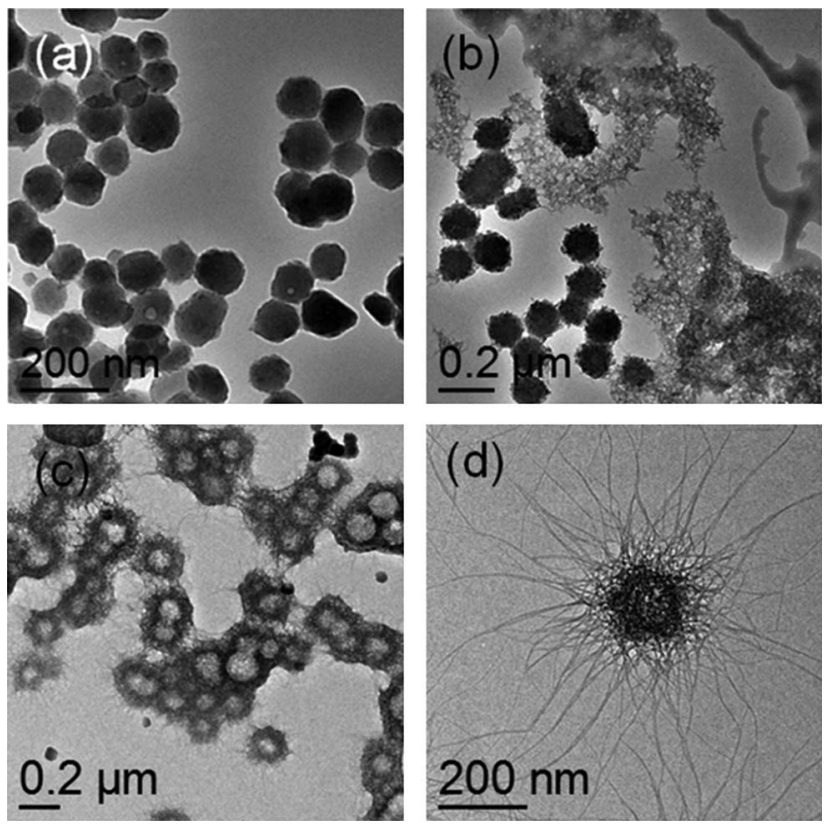

Fig. 3 Morphological sequence of events that is believed to take place during development of the neuron-like structures. Synthesis conditions: $[\mathrm{Ca}]=[\mathrm{P}]_{\text {final }}=5 \mathrm{mM}$, TW content $0.01 \mathrm{wt} \%$ (a) formation of hybrid organic/inorganic clusters of amorphous precursors, (b) secondary nucleation of branches taking place on the surface of the amorphous structures, (c) further growth of the branches controlled by the diffusion of reactants and (d) a fully developed structure. Note that the hollow sphere shown in (c) is caused by excessive irradiation of the amorphous core with the electron beam. Once the whole structure is developed no irradiation damage was observed.

(Fig. 4). The change in the conductivity profile was predictable as raising the temperature accelerates crystallization. In fact, at $60{ }^{\circ} \mathrm{C}$ it was not possible to observe the amorphous regime anymore but, as mentioned, this did not hamper the stabilization of the amorphous neuron-like structures. The fact that the amorphous regime could not be detected simply meant that the time frame it existed was too short to be visualized. One aspect that should be borne in mind in all the above experiments is that in spite of the remarkable structures that TW can form, bulk crystallization does still take place and the coexistence of the stabilized amorphous neuron-like structure along with the crystallized phase complicates the interpretation of results.

The close similarity between the neuron-like structures obtained with this simple surfactant and those discovered by Antonietti et al. ${ }^{20}$ using DHBCs clearly prove that such structures are not specific of the complex copolymer. To further reinforce this aspect, additional experiments were performed using a different surfactant. Span 20 (sorbitan monolaurate, SP), a non-ionic surfactant of smaller size and even lesser complexity than TW, was chosen for this purpose. Fig. 5 summarizes the conductivity profiles obtained by adding different amounts of SP and the corresponding micrographs. Although the deep effect that this particular surfactant has on the amorphous to crystalline transition is striking (conductivity profiles), more surprising is the fact that different surfactants (TW, SP, and DHBCs), with markedly different conductivity 

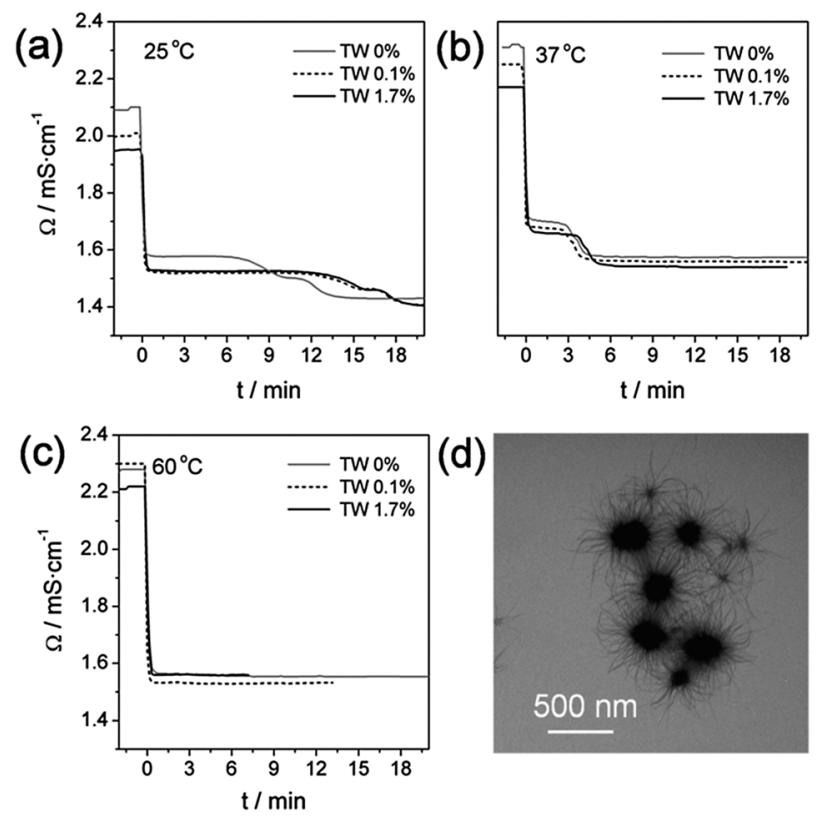

Fig. 4 From (a to c) conductivity profiles for synthesis in the presence of various contents of TW $(0,0.1$ and $1.7 \mathrm{wt} \%)$ at various temperatures: (a) $25^{\circ} \mathrm{C}$, (b) $37{ }^{\circ} \mathrm{C}$, and (c) $60^{\circ} \mathrm{C}$. (d) TEM image of the resulting precipitate for a synthesis performed at $60{ }^{\circ} \mathrm{C}$ with a $0.1 \mathrm{wt} \% \mathrm{TW}$.

profiles (TW versus SP), can form identical type of complex neuron-like structure. The two types of CaP structures that SP forms and the overall delay observed in the conductivity profiles
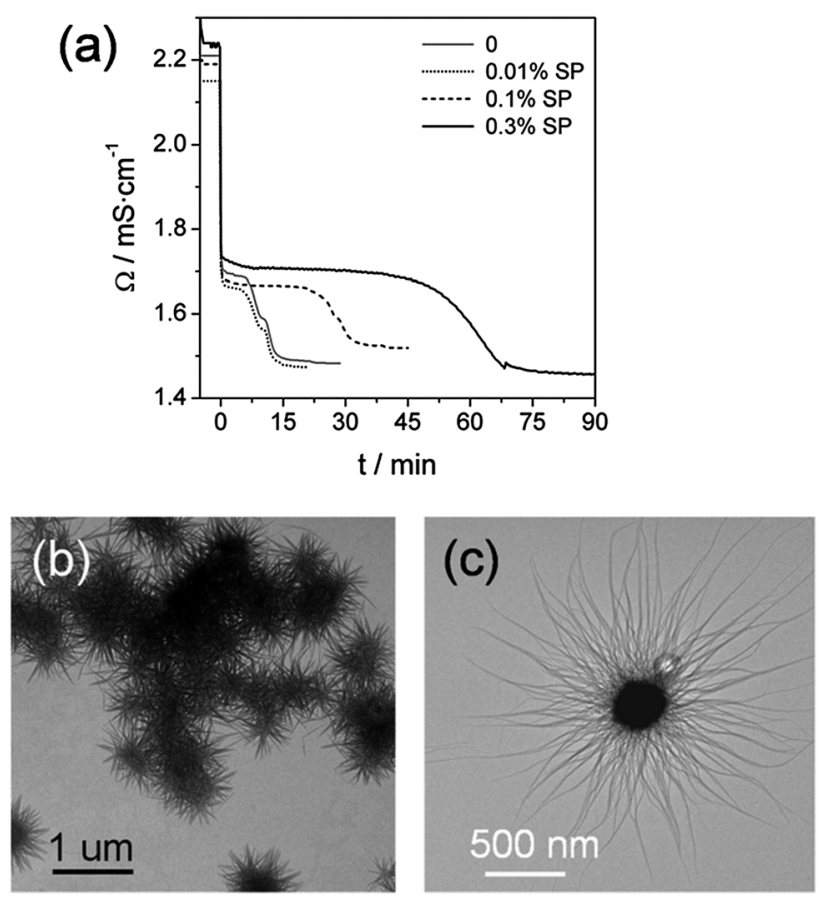

Fig. 5 (a) Effect of the addition of various contents of Span 20 (SP) on the conductivity profile. SP was first allowed to mix with the calcium chloride solution before the phosphate solution was added at time zero. The concentrations of the salts were identical as in Fig. 1(b) and (c) TEM images for a SP content of 0.1 wt $\%$ after 5 days. The TEM grids were prepared from the supernatant (the large crystallized product was concentrated in the sediment). have to be regarded with care as SP, unlike TW, is a more hydrophobic molecule and this could affect the results. This however did not impede formation of the neuron-like structures as proved in Fig. 5 .

One important question that may arise when looking at these neuron-like structures is whether they are real or they could be artifacts formed during drying of the TEM grid. In the work of Antonietti et al., analytical ultracentrifugation (AUC) studies were performed to prove their existence. ${ }^{20}$ In our case, field emission scanning electron microscopy (FE-SEM) was used to further corroborate their existence. Our hypothesis was that if these structures were artifacts, they would result from the assembly of filaments deposited on the grid upon drying, and they should form 2D structures, however, if the neuron-like structures would be real, filaments would be surrounding the whole structure forming a 3D assembly. Fig. 6 clearly shows the $3 \mathrm{D}$ core of the structure and though filaments could not be seen standing up from the core, this can be caused by the fact that the flexible filaments stick to the structure upon drying.

\subsection{Calcium phosphate precipitation in the presence of sodium polyacrylate}

Antonietti et al. proposed that the mechanism of formation of the neuron-like structures was tightly linked to the amphiphilic character of the organic molecule (DHBC- $\mathrm{C}_{12}$ ) ${ }^{20}$ They observed that when the hydrophobic contribution $\left(\mathrm{C}_{12}\right.$ alkanes) was not present in the DHBC, the neuron-like structures could not form. The hydrophobic moieties were suggested to induce aggregation of the polymer. This simple step was however crucial as was believed to allow sequestration/concentration of calcium ions thus serving as localized mineralization centers. A similar scenario might occur when using surfactants like TW or SP where the hydrophilic head (e.g. that of a micelle or even that of a single molecule), rich in oxygen and thus carrying a negative density owing to the presence of electron pairs, can concentrate calcium ions and induce mineralization. The remarkably high anisotropy of the filaments suggests a strong interaction between the organic molecule and the mineral phase in the

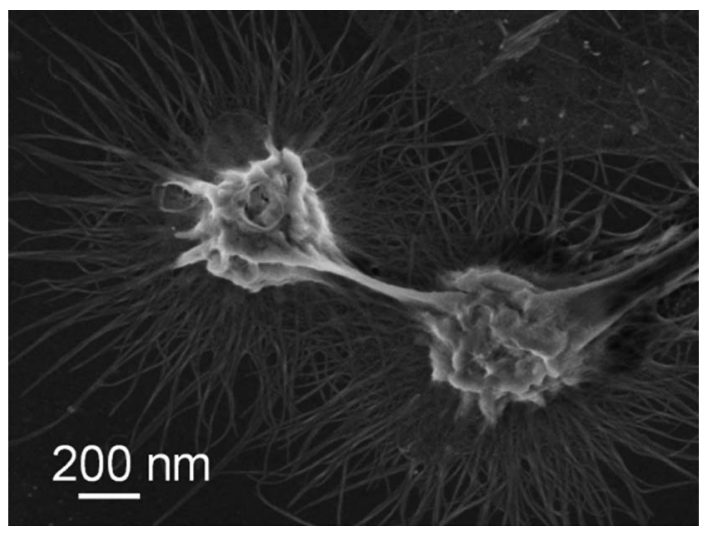

Fig. 6 FE-SEM image of typical neuron-like structures viewed directly from a TEM grid without any preparation. $[\mathrm{Ca}]=[\mathrm{P}]_{\text {final }}=5 \mathrm{mM}$, TW content $0.1 \mathrm{wt} \%$. 
formation of the neuron-like structures ${ }^{24}$ but the exact mechanism of formation still remains unclear. To prove that indeed the formation of the neuron-like structures requires an amphiphilic molecule, sodium polyacrylate, a widely used synthetic polymer of anionic nature, ${ }^{25-29}$ was introduced in the calcium phosphate precipitation system to replace the surfactant molecule. Presumably, with such a molecule, the neuronlike structures should not form as they lack hydrophobic moieties. For this purpose a $0.06 \mathrm{wt} \%$ of sodium polyacrylate (PA) was first mixed with a $10 \mathrm{mM} \mathrm{CaCl}_{2}$ solution onto which, an equal volume of $10 \mathrm{mM} \mathrm{Na} \mathrm{HPO}_{4}$ with $\mathrm{pH}$ adjusted to a physiological value was added. Such reaction conditions were identical to those used for the TW and SP system and served to highlight the strong impact that PA has on the nucleation of calcium phosphate. In the first place, the addition of a total of $0.03 \mathrm{wt} \%$ of PA into the precipitating system impeded the bulk precipitation of calcium phosphate crystals as was macroscopically observed from the clear solutions. This was also confirmed from the conductivity profile where no ACP to crystalline transition could be detected (Fig. 7a). The reason for the inhibition of crystal formation is explained by the higher density of negative charge in the PA molecule when compared to that in the surfactant molecules which results in binding of many more calcium ions to the organic molecule thus impeding their contribution towards bulk crystal precipitation. A TEM grid was prepared to prove whether any precipitate was formed at all and the results showed the presence of an entangled network of mineralized filaments throughout (Fig. 7b).

Analyses of the filaments by EDX revealed that they contained $\mathrm{Ca}$ and $\mathrm{P}$ at a $\mathrm{Ca} / \mathrm{P}$ ratio of $\sim 1$. Such findings seemed to prove that indeed the lack of hydrophobic moieties could not stabilize the neuron-like structures but the striking similarity of these filament networks to the neuron-like structures suggests that the former could simply be an immature state of the latter (or vice versa). Thus, to verify this point, several additional experiments were performed adjusting the reaction conditions. It was observed that the increase in the concentration of reagents while keeping $\mathrm{Ca} / \mathrm{PA}$ and $\mathrm{Ca} / \mathrm{P}$ ratios constant seemed to favor the formation of the neuron-like structures (Fig. 8) but it was the increase in the $\mathrm{Ca} / \mathrm{PA}$ ratio for a constant calcium concentration

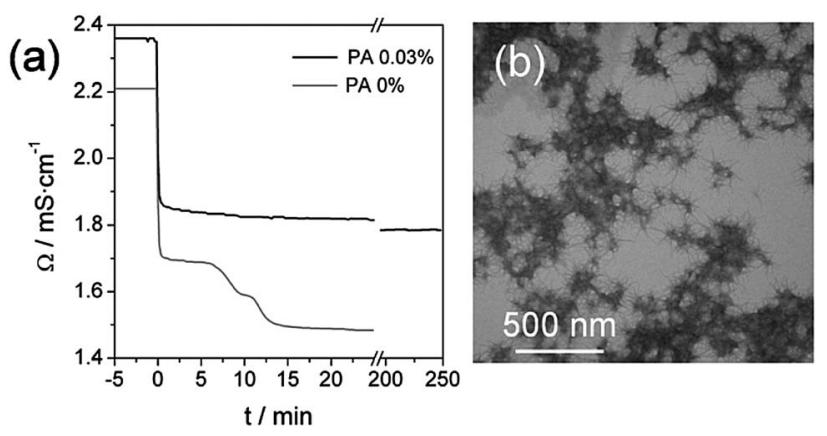

Fig. 7 (a) Effect of the addition of sodium polyacrylate (PA) on the conductivity profile. PA was first allowed to mix with the calcium chloride solution before the phosphate solution was added at time zero. The concentrations of the salts were identical as in Fig. 1(b) TEM image resulting from the PA synthesis.
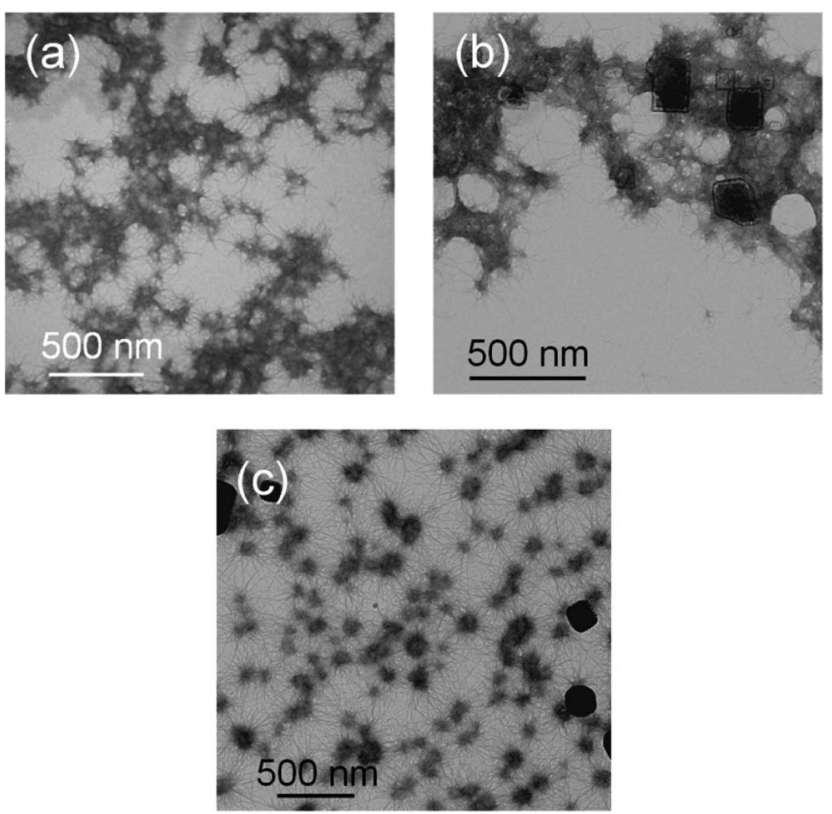

Fig. 8 TEM images showing the effect of varying the synthesis conditions (salt concentration) on the microstructure of the precipitates. (a) $[\mathrm{Ca}]_{\text {final }}=5 \mathrm{mM}, \mathrm{Ca} / \mathrm{PA}=20$ and $\mathrm{Ca} / \mathrm{P}=1.5$, (b) $[\mathrm{Ca}]_{\text {final }} \sim 10$ $\mathrm{mM}, \mathrm{Ca} / \mathrm{PA}=20$ and $\mathrm{Ca} / \mathrm{P}=1.67$, and (c) $[\mathrm{Ca}]_{\text {final }} \sim 50 \mathrm{mM}, \mathrm{Ca} / \mathrm{PA}=20$ and $\mathrm{Ca} / \mathrm{P}=1.67$. Samples presenting bulk $\mathrm{CaP}$ crystal precipitation (b and c) were allowed to sediment prior to TEM grid preparation.

and $\mathrm{Ca} / \mathrm{P}$ ratio that was critical in their stabilization (Fig. 9). We believe that this increase in the $\mathrm{Ca} / \mathrm{PA}$ ratio (which implies decreasing the PA content) favors the stabilization of the typically looking "ACP" globular clusters of hybrid nature which are in essence the central core of the neuron-like structures. When these globular clusters become smaller (this is favored at higher PA contents) a few filaments from each tiny core (see e.g. Fig. 9a and b) instead of the well-formed neuron-like structures are generated. Overall these are important results as they prove that organic molecules other than surfactants work alike. The question that follows is whether the hydrophobic moiety, so indispensable in the work of Antonietti et al., is indeed necessary for the stabilization of such structures? Apparently it may seem that the answer is no, but this would not be entirely true because upon binding of the calcium ions to the carboxylic groups from PA, neutralization of charges occurs thus rendering the molecule with a more hydrophobic character and causing their aggregation. ${ }^{30}$ This reasoning was used by Tjandra et al. to justify the formation of neuron-like structures with a DHBC that lacked a specifically hydrophobic block. ${ }^{21}$ Moreover, the same explanation might serve to justify why in the work of Peytcheva et al. polyaspartate was also capable of forming such structures. ${ }^{22}$

One interesting aspect when working with ionic molecules such as PA is that tuning the $\mathrm{pH}$ causes different degrees of protonation of the carboxylic groups thus altering the binding ability towards calcium. This effect has been investigated with regard to the formation of neuron-like structures and the results are summarized in Fig. 10 and 11. Fig. 10a represents the graphs obtained after the titration of a $10 \mathrm{mM}$ calcium chloride with a $10 \mathrm{mM}$ sodium polyacrylate solution (1 wt\%) added 

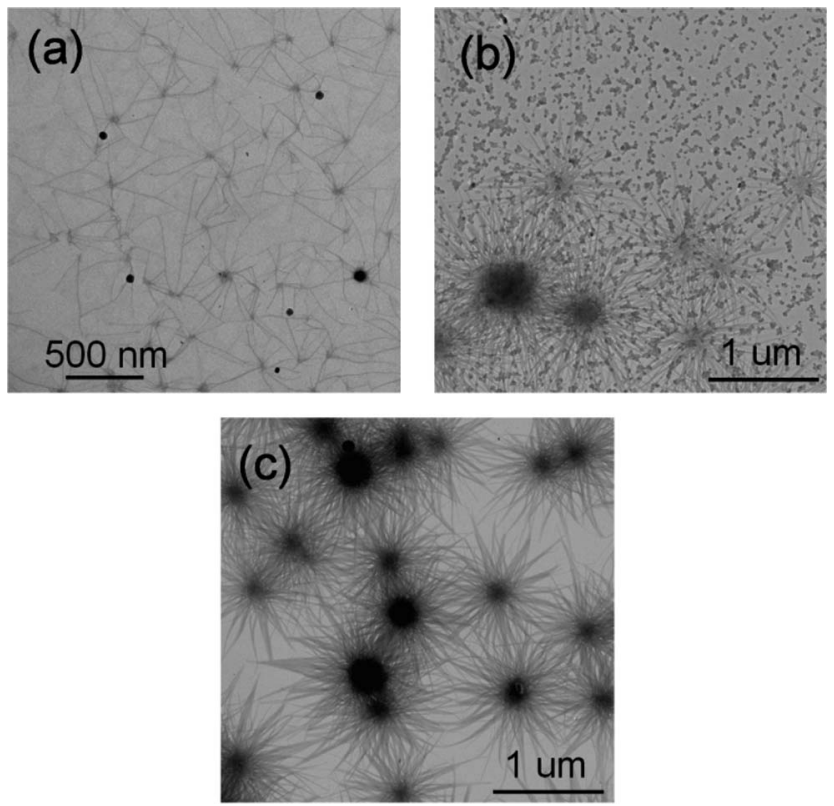

Fig. 9 TEM images showing the effect on the microstructure of increasing the $\mathrm{Ca} / \mathrm{PA}$ ratio to 80 . Other synthesis conditions were: $[\mathrm{Ca}]_{\text {final }} \sim 15 \mathrm{mM}$ and $\mathrm{Ca} / \mathrm{P}=1.67$. All these different types of microstructures $(a-c)$ coexisted: fiber-like structures, small rounded aggregates and neuron-like structures.

droplet by droplet into the system at a rate of $0.5 \mathrm{ml} \mathrm{min}{ }^{-1}$. The $\mathrm{pH}$ of both solutions was previously adjusted to: 3,7 and 10. As can be observed from the $\mathrm{pH} 3$ curve, since the moment of addition of PA (time zero), the conductivity of the system rises gradually indicating that there is no interaction between the protonated carboxylic groups with calcium. At pH 7 and 10, the scenario changes as observed from the curves obtained. At the beginning, the addition of PA does not seem to alter the conductivity of the system and only after a specific time it starts rising. This apparently unperturbed behavior at the beginning of the titration is an indication that the de-protonated carboxylic groups are binding calcium ions thus they do not contribute to the increase of the conductivity of the system. After a specific time, however, the conductivity begins to increase indicating that all de-protonated carboxylic groups have already been calcium bound. Surprisingly, no significant differences were detected when comparing the profiles run at pH 7 and 10. We believe however that this might be caused by the design of the experiment. The addition of PA droplet by droplet onto a calcium solution results in a huge amount of calcium ions that may force a fast coiling of the PA burying some unreacted carboxylic groups within the coiled structure. By simply performing the experiment the other way round, i.e. by adding calcium ions droplet by droplet into the PA solution (Fig. 10b), this problem is solved and as can be judged from Fig. 10b that the profiles now reveal what one would have expected: that the fully de-protonated PA at $\mathrm{pH} 10$ would be more efficient at neutralizing the calcium in solution than at $\mathrm{pH} 7$.

To validate if these different scenarios affected the formation of the neuron-like structures, TEM grids were prepared by mixing $\mathrm{Ca}+\mathrm{PA}$ with $\mathrm{Na}_{2} \mathrm{HPO}_{4}$ at various $\mathrm{pH}$. Regardless of the
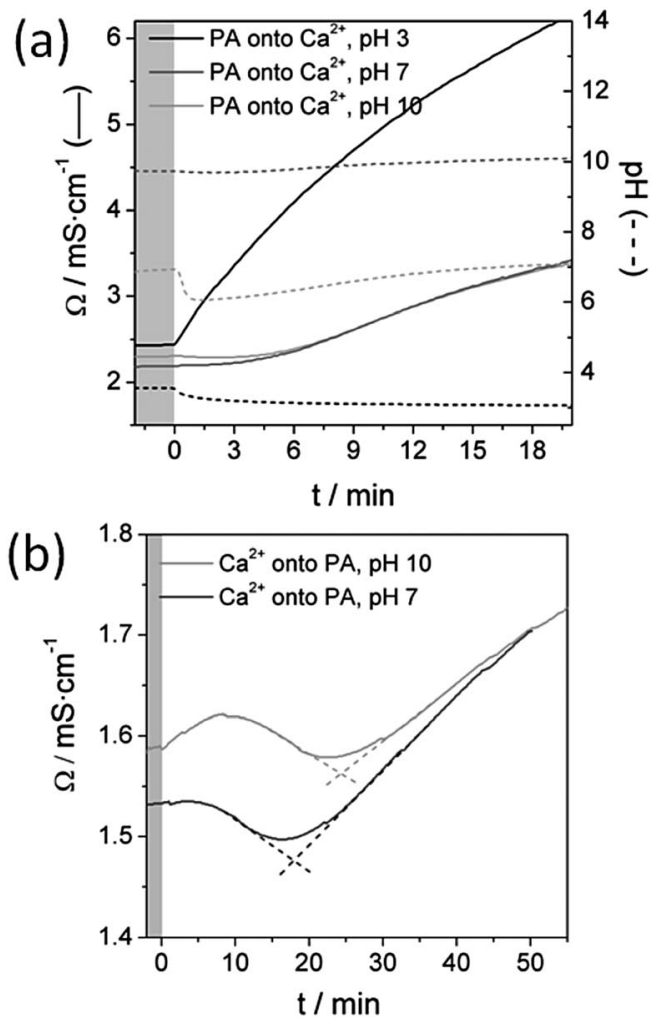

Fig. 10 Changes in conductivity during titration of (a) $10 \mathrm{mM} \mathrm{PA}$ added at a $0.5 \mathrm{ml} \mathrm{min}-1$ rate into $15 \mathrm{ml}$ of $10 \mathrm{mM} \mathrm{CaCl}_{2}$ solution at various $\mathrm{pH}$ : 3,7 and 10 , and (b) titration profile of $10 \mathrm{mM} \mathrm{CaCl}_{2}$ added at a $0.5 \mathrm{ml}$ $\mathrm{min}^{-1}$ rate into $15 \mathrm{ml}$ of $2 \mathrm{mM} \mathrm{PA}$ at $\mathrm{pH} 7$ and 10 . The intersection points shown in (b) indicate the Ca/PA ratio needed to neutralize the carboxylic groups available in $\mathrm{PA}(\mathrm{Ca} / \mathrm{PA}=4$ at $\mathrm{pH} 10$ and $\mathrm{Ca} / \mathrm{PA}=3$ at $\mathrm{pH}$ 7).
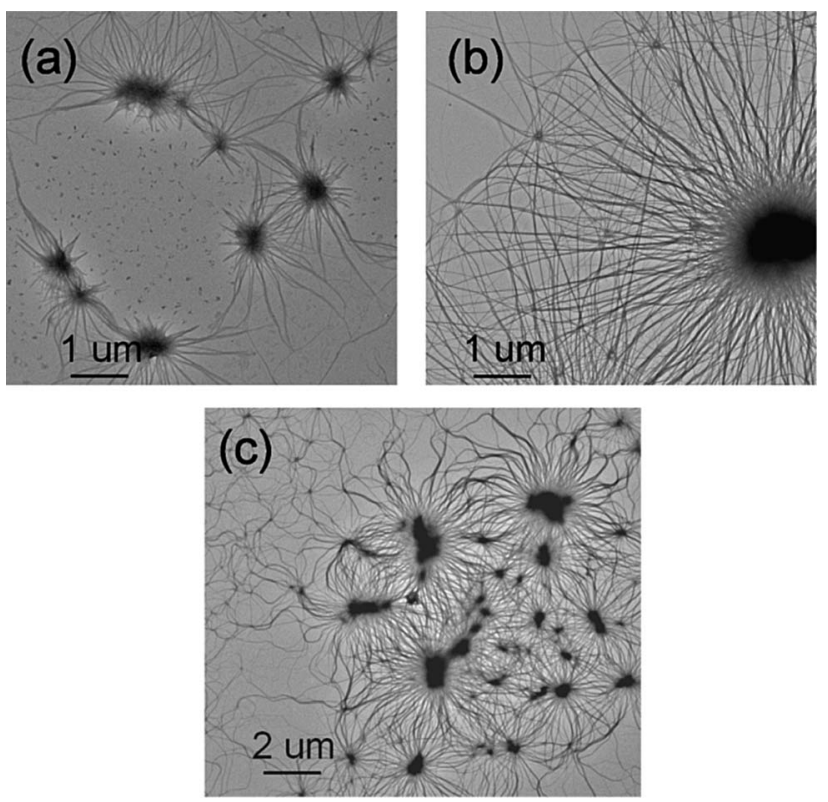

Fig. 11 TEM images obtained from synthesis reactions in the presence of $\mathrm{PA}$ at $\mathrm{pH} 3$ and $\mathrm{Ca} / \mathrm{PA}=80$. (a) $[\mathrm{Ca}]_{\text {final }} \sim 5 \mathrm{mM}$, (b) $[\mathrm{Ca}]_{\text {final }} \sim 15 \mathrm{mM}$ and (c) $[\mathrm{Ca}]_{\text {final }} \sim 50 \mathrm{mM}$. Ca/P $=1.67$. Although figure $(\mathrm{a})$ is representative of all conditions, other interesting microstructure has been selected in conditions (b) and (c). 
$\mathrm{Ca} / \mathrm{PA}$ ratio and the concentration of reactants, the $\mathrm{pH}$ of the reaction had a strong impact on the formation of the neuronlike structures. Working at pH 3 macroscopically yielded clear solutions (with no bulk CaP precipitation) but microscopically revealed the presence of the typical neuron-like structures of various sizes along with the formation of long twisted bundles as can be observed from Fig. 11c. At first sight this result might seem surprising as one would expect that without interaction of the calcium ions with the protonated carboxylic groups in PA, there should not be formation of the neuron-like structures. However we already proved that with organic molecules with non-bearing charges (anionic surfactants) it was already possible to form them. Thus working at $\mathrm{pH} 3$ simply confirms that the formation of the neuron-like structures can take place via weak electrostatic forces of ion-dipole $\left(\mathrm{Ca}^{2+}-\mathrm{COOH}\right)$ nature and not necessarily through ion-ion bonding $\left(\mathrm{Ca}^{2+}-\mathrm{COO}^{-}\right)$. In fact, in the work of Antonietti et al., they also showed that working at $\mathrm{pH} 3 \mathrm{did}$ not hamper the formation of such structures.

At $\mathrm{pH} 10$, and at a calcium concentration low enough to minimize $\mathrm{Ca}(\mathrm{OH})_{2}$ precipitation, there was no formation of the neuron-like structures and, instead, the bulk precipitation of hydroxyapatite (HA) was observed. Macroscopically this $\mathrm{pH}$ condition yielded an opaque solution evidencing HA crystallization. Working at neutral $\mathrm{pH}$ gave an intermediate condition in which the neuron-like structures coexisted with other amorphous structures. The apparently contradictory result from the lack of neuron-like structures in a condition where most of the carboxylic groups of PA were de-protonated (high $\mathrm{pH}$ ), and therefore bound to calcium, can be explained by a too strong driving force towards HA precipitation at this particular $\mathrm{pH} .{ }^{20}$ The continuous formation of HA crystals would lead to the gradual depletion of the calcium bound to PA to further contribute to HA precipitation.

The various experiments performed with PA as growth directing unit have proven that such a molecule can form identical neuron-like structures than those obtained with the help of surfactants but with the additional advantage that under suitable adjustment of the reaction conditions the product could be made practically pure. Although the mechanism of formation is not totally understood, the results with PA evidence that the need for working with organic amphiphilic molecules (hydrophobic-hydrophilic) is not compulsory and the formation of the neuron-like structures is rather a generic phenomenon. Whether this apparently simple mechanism of formation is particular of the cation/organic molecule system or not will be explored in this next section by investigating the feasibility of inducing similar structures with the help of a cationic polymer. The goal would thus be to prove if the cationic polymer is capable of directing anion binding (phosphate group) towards formation of the neuron-like structures.

\subsection{Calcium phosphate precipitation in the presence of polydiallyldimethylammonium chloride}

As the cationic polymer polydiallyldimethylammonium chloride (PDMAC), a strong polyelectrolyte, was chosen for this particular study. Being a strong polyelectrolyte the charge of this polymer is not affected by the $\mathrm{pH}$; thus, all experiments were done at one $\mathrm{pH}$ condition only, i.e. close to neutrality. In a typical synthesis reaction PDMAC was first mixed with the phosphate salt to facilitate interaction of the anion with the molecule and then calcium chloride was added to precipitate calcium phosphate. Two different concentrations of PDMAC, 0.1 and $0.001 \mathrm{wt} \%$, were explored. The concentration of the calcium and phosphate solutions used was the same as those chosen in the PA experiments, i.e. $\left[\mathrm{Ca}^{2+}\right]_{\text {final }}=50 \mathrm{mM}$ and $[\mathrm{P}]_{\text {final }}$ $=30 \mathrm{mM}$, to allow comparison of results. These high concentrations were purposely selected to favor neutralization (by charge screening) of the amine groups from the polyelectrolyte and induce coiling/aggregation of the polymer. The coiled structure should then act as concentrated phosphate ions that would serve as a nucleation center in the formation of the neuron-like structures. Regardless of the content of PDMAC the synthesis reaction followed the profile shown in Fig. 12a in which amorphous and crystallized phases could be distinguished (refer to the $\mathrm{pH}$ profile). The most important fact was, however, that even after crystallization took place, amorphous structures resembling the neuron-like structures were also formed in this particular setup (Fig. 12). It should be mentioned, however, that with this particular polymer the structures were not stable and after a few days they disappeared.

The results thus far clearly demonstrate that formation of the neuron-like structures is governed by a generic mechanism in which the organic molecule serves as a substrate for $\mathrm{CaP}$ precipitation and at the same time directs the growth/assembly towards the final structure. Whether the organic molecule is
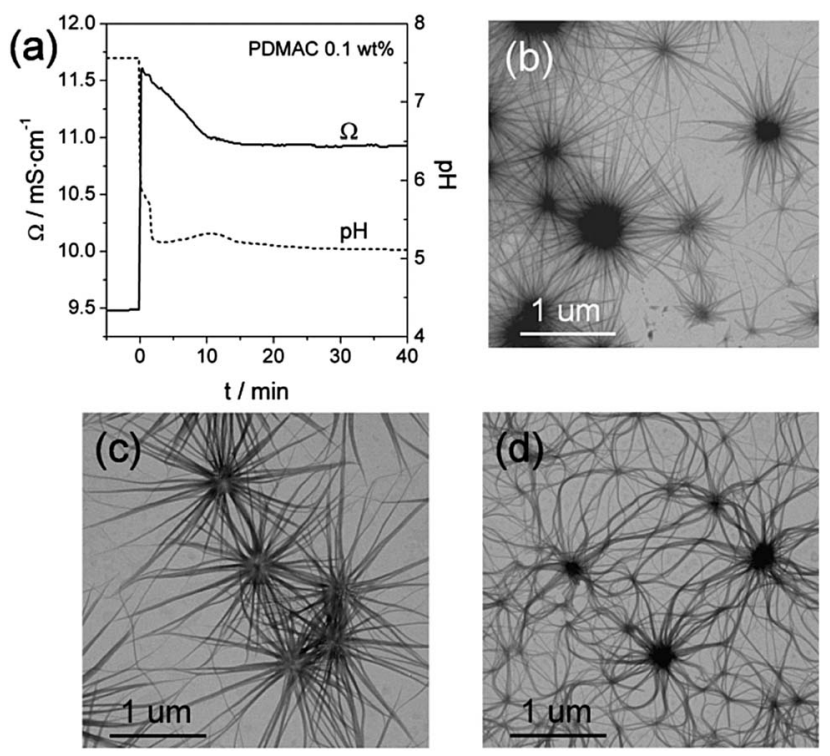

Fig. 12 (a) Effect of the addition of polydiallyldimethylamino chloride (PDMAC) on the conductivity and $\mathrm{pH}$ profile. PDMAC was first allowed to mix with the phosphate solution before the calcium chloride solution was added at time zero. TEM images of structures stabilized with 0.1 (b) and 0.001 wt\% (c and d) of PDMAC. $\mathrm{Ca} / \mathrm{P}=1.67$ and $[\mathrm{Ca}]_{\text {final }}$ $\sim 50 \mathrm{mM}$. The TEM grids were prepared from the supernatant (the large crystallized product was concentrated in the sediment). 
negatively or positively charged or does not have any charge at all does not seem to be critical in the stabilization of the structures as in all cases the neuron-like structure can form. An interesting aspect to note is that in spite of the high anisotropy of the filaments, which underlines a tight interaction between the organic molecule and the inorganic phase, these structures can form through weak electrostatic forces as was confirmed in the experiments with non-ionic surfactants and also PA at $\mathrm{pH} 3$. Moreover, we have also proved that such structures can be stabilized regardless of whether the polymer associates first with the anion (PDMAC with the phosphate ion) and then the cation is added, or vice versa (PA with the calcium ion). But in spite of their facile formation not all conditions allow their stabilization. Insufficient levels of calcium and phosphate can only stabilize a filamentous structure (Fig. 7b) and a too high content of the organic molecule adversely affects their formation by quenching the mineralized nuclei and impeding their subsequent assembly (Fig. 2c). The need for high ionic strengths when dealing with polyelectrolytes (PA and PDMAC), we believe, is to ensure coiling of the molecule since in environments of low ionic strength the charged areas on the polymer chain repel each other and force the chain to uncoil. ${ }^{\mathbf{3 1 , 3 2}}$ Instead, at high ionic strengths the small ions screen the charges from the polymer causing it to coil. Thus, the high content of calcium and phosphate ions required for the neuronlike formation in the case of PA and PDMAC seems to agree well with the fact that the polymer needs to cluster/aggregate first prior to their formation but any further insight into the full development of the final structure is out of the scope of the work.

The outcome of the present work becomes particularly interesting from two points of view. On the one hand, the closeness of the synthesis reactions, in terms of reaction conditions and the nature of reactants to those reactions occurring in natural systems, makes the present results very valuable for the interpretation of biomineralisation events and can shed new light on their formation. On the other hand, regardless of whether nature can form such structures or not, they could be of potential interest in other disciplines and the concepts derived from the present work can be applied to synthesize nanoparticles with particular properties. Although more work remains to be done to fully understand the mechanism of formation of the neuron-like structures, we believe that a very generic mechanism involving weak electrostatic forces is governing the formation of such structures and probably many other molecules of even different nature can work alike.

\section{Conclusions}

This work serves to prove that the formation of calcium phosphate neuron-like structures occurs through a more generic mechanism than the one established with the help of complex double hydrophilic block copolymers. Identical structures have been formed using simple amphiphilic molecules of non-ionic nature (Tween 80 and Span 20), anionic polymers (sodium polyacrylate) and cationic polymers (polydiallyldimethylammonium chloride) combined with calcium and phosphate salts under nearly ambient conditions. In spite of the high anisotropy of the resulting structures which accounts for a tight interdigitation between the organic and inorganic phases, such structures can be stabilized by weak electrostatic forces of ion-dipole nature. The reaction conditions were found to be critical in stabilizing this particular type of structure and determined the purity of the product. Within the range of conditions investigated, only with the addition of PA and under specific conditions, the neuron-like structure could be made practically pure. In all other cases, the formation of these structures competed with the precipitation of CaP crystals. The facile stabilization of such structures under ambient conditions makes them potential candidates for biomineralisation reactions and also offers the means of synthesizing a new class of structures with additional properties.

\section{Acknowledgements}

The authors are very grateful to Dr Isidre Casals for fruitful discussions and to Dr Trifon Trifonov for his help in the FE-SEM observation. This study was supported by the Spanish Ministry of Economy and Competitiveness (MINECO) through the project: MAT2012-38438-003-01. Support for the research of MPG was received through the ICREA Academia prize for excellence in research, funded by the Generalitat de Catalunya.

\section{Notes and references}

1 S. Weiner and P. M. Dove, An Overview of Biomineralisation Processes and the Problem of the Vital Effect, ed. P. M. Dove, J. J. DeYoreo and S. Weiner, Washington, DC, 2003, pp. 1-29. 2 F. C. Meldrum and H. Colfen, Chem. Rev., 2008, 108, 4332.

3 Y. Kakizawa, K. Miyata, S. Furukawa and K. Kataoka, $A d v$. Mater., 2004, 16, 699.

4 H. Cölfen and M. Antonietti, Polymer-Controlled Biomimetic Mineralization of Novel Inorganic Materials, ed. A. Sigel, $\mathrm{H}$. Sigel and R. K. O. Sigel, John Wiley \& Sons, Chichester, 2010, pp. 607-643.

5 P. Y. Chen, J. McKittrick and M. A. Meyers, Prog. Mater. Sci., 2012, 57, 1492.

6 J. Mahamid, A. Sharir, L. Addadi and S. Weiner, Proc. Natl. Acad. Sci. U. S. A., 2008, 105, 12748.

7 E. Beniash, R. A. Metzler, R. S. K. Lam and P. U. P. A. Gilbert, J. Struct. Biol., 2009, 166, 133.

8 C. Combes and C. Rey, Acta Biomater., 2010, 6, 3362.

9 M. Grynpas and S. Omelon, Bone, 2007, 41, 162.

10 L. B. Gower, Chem. Rev., 2008, 108, 4551-4627.

11 B. J. Ofir, R. Govrin-Lippman, N. Garti and H. FurediMilhofer, Cryst. Growth Des., 2004, 4, 177.

12 M. D. Sikirić and H. Füredi-Milhofer, Adv. Colloid Interface Sci., 2006, 128-130, 135.

13 K. Bleek and A. Taubert, Acta Biomater., 2013, 9, 6283.

14 R. Q. Song and H. Colfen, CrystEngComm, 2011, 13, 1249.

15 C. E. Fowler, M. Li, S. Mann and H. C. Margolis, J. Mater. Chem., 2005, 15, 3317.

16 R. Q. Song, H. Cölfen, A. W. Xu, J. Hartmann and M. Antonietti, ACS Nano, 2009, 3, 1966. 
17 H. Cölfen and L. Qi, Chem. - Eur. J., 2001, 7, 106.

18 L. Qi, H. Cölfen, M. Antonietti, M. Li, J. D. Hopwood, A. J. Ashley and S. Mann, Chem. - Eur. J., 2001, 7, 3526.

19 A. W. Xu, Y. Ma and H. Colfen, J. Mater. Chem., 2007, 17, 415.

20 M. Antonietti, M. Breulmann, C. G. Göltner, H. Cölfen, K. W. K. Wong, D. Walsh and S. Mann, Chem. - Eur. J., 1998, 4, 2493.

21 W. Tjandra, J. Yao, P. Ravi, K. C. Tam and A. Alamsjah, Chem. Mater., 2005, 17, 4865.

22 A. Peytcheva, H. Cölfen, H. Schnablegger and M. Antonietti, Colloid Polym. Sci., 2002, 280, 218.

23 E. D. Eanes, J. D. Termine and M. U. Nylen, Calcif. Tissue Res., 1973, 12, 143.

24 S. Mann, Angew. Chem., Int. Ed., 2000, 39, 3392.
25 S. Yu, M. Antonietti and H. Cölfen, Nano Lett., 2003, 3, 379.

26 S. C. Liou, S. Y. Chen and D. M. Liu, J. Biomed. Mater. Res., Part B, 2005, 73, 117.

27 T. Wang and H. Cölfen, Langmuir, 2006, 22, 8975.

28 A. Bigi, E. Boanini, M. Gazzano and A. Kojdecki, J. Mater. Chem., 2004, 14, 274.

29 A. Bigi, E. Boanini, D. Walsh and S. Mann, Angew. Chem., Int. Ed., 2002, 41, 2163.

30 R. Schweins and K. Huber, Eur. Phys. J. E: Soft Matter Biol. Phys., 2001, 126, 117.

31 Z. Adamczyk, A. Bratek, B. Jachimska, T. Jasiński and P. Warszyński, J. Phys. Chem. B, 2006, 110, 22426.

32 B. Jachimska, T. Jasiński, P. Warszyński and Z. Adamczyk, Colloids Surf., A, 2010, 355, 7. 